\title{
Ecological conditions for Saxifraga hirculus in Central Europe: a better understanding for a good protection
}

\author{
Pascal Vittoz ${ }^{1}$, Jean-Michel Gobat ${ }^{2}$, Tania Wyss ${ }^{1}$ \\ ${ }^{1}$ University of Lausanne, Department of Ecology and Evolution (Faculty of Biology and \\ Medicine) and Faculty of Earth Sciences and Environment, Bâtiment Biophore, 1015 \\ Lausanne, Switzerland. \\ ${ }^{2}$ University of Neuchâtel, Institute of Botany, Laboratory "Soil \& Vegetation”, Rue Emile- \\ Argand 11, 2000 Neuchâtel, Switzerland. \\ Corresponding author: Pascal Vittoz, tel: +41-21-692-4270; fax: +41-21-692-4265, e-mail: \\ pascal.vittoz@.unil.ch
}

\begin{abstract}
Saxifraga hirculus is a postglacial relict in Central Europe, whose populations suffered a dramatic decrease in the $19^{\text {th }}$ and $20^{\text {th }}$ centuries. However, few researchers have been interested in its ecological requirements in Central Europe. This article synthesizes previous knowledge supplemented by original data from the last large population (Switzerland).

$S$. hirculus is a weak competitor which needs precise ecological conditions. It grows on bryophyte carpets in neutral to slightly acid wetlands, with stable water table close to the soil surface (optimum between 8-14 cm) but does not stand long flooding. However, it requires a good oxygen supply, with roots $2-3 \mathrm{~cm}$ under the soil surface, generally not reached by water, with running, cold water through loose, fibric peat. Its optimal conditions are in spring fens, but it grows in other types of wetlands as well. However, overgrowing by shrubs, sedges or Sphagnum in natural successions may threaten the species with extinction, as did drainage and peat extraction previously. Now, its survival in Central Europe depends on an adequate management of the ecosystems. Moderate grazing (cattle or sheep) or mowing help to limit competition with taller Carex species. Re-introduction of disappeared populations or creation of new ones from cultivation in botanical garden is possible, but appropriate sites are rare. In some cases, substrate management could improve the conditions in somewhat inadequate situations. This management in four directions can be flexibly applied in different situations to progress to optimal conditions for the conservation of this valuable species.
\end{abstract}

Keywords: cattle grazing, ecosystem management, Jura Mountains, wetland conservation, soil description.

Nomenclature: Tutin et al. (1964-1980) for plants, Frahm and Frey (1983) for bryophytes, and AFES (1998) for soils. 


\section{Introduction}

Saxifraga hirculus L. is a perennial herbaceous plant of mires and various types of tundra. It has an important clonal reproduction with leafy runners able to root (Olesen and Warncke, 1990). Pollinisation of flowers is realized by a large spectrum of insects, mainly Diptera (Olesen and Warncke, 1989; Warncke et al., 1993). It has a circumpolar distribution, with a Central Asiatic origin and an Arctic-Boreal distribution (Hedberg, 1992). Four subspecies have been described around the world, with chromosome numbers of $2 n=16,24$ or 32 (Hedberg, 1992). In continental Europe, only S. hirculus subsp. hirculus $(2 \mathrm{n}=32)$ is present, with an scattered distribution (Fig. 1). However, it is very rare and endangered in Central and Western Europe, and rare in Scandinavia (Käsermann and Moser, 1999). Five stands exist in Ireland (Lockart, 1989), five in Scotland (Welch, 2002 and pers. comm.), it is very restricted in England but with large populations (NBN Gateway), there are 15 stands in Denmark (Dahlgaard and Warncke, 1994), about 100 in Poland, mainly in north, considered as vulnerable in Rumania (Boscaiu et al., 1994), two stands in France, with less than 100 plants altogether (Dehondt and Ferrez, 2004), and one in Switzerland with more than 10000 flowers in 1992 (Warncke et al., 1993). The species is now protected by the European Habitats Directive and the Bern Convention.

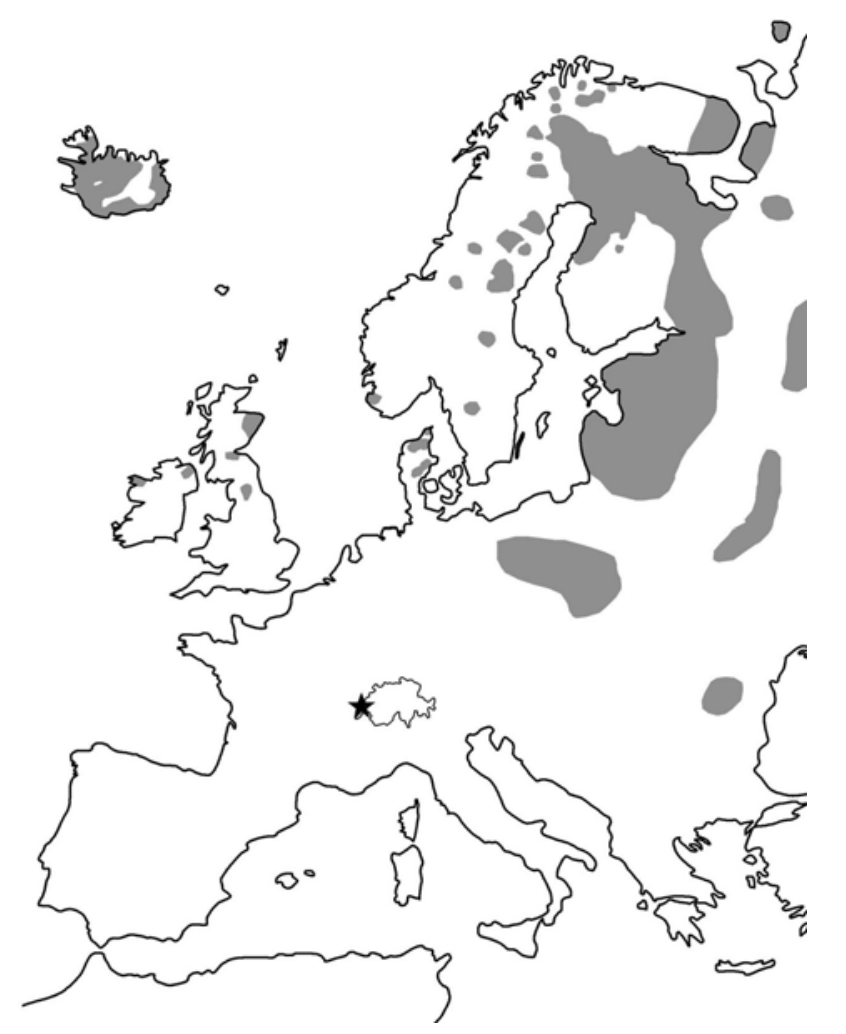

Fig. 1. Distribution map of Saxifraga hirculus L. in Europe following Hedberg (1992) for Romania and NorthEastern Europe, Stenberg and Mossberg (2003) for Scandinavia and Iceland, NBN Gateway for United Kingdom. $\star$ Localization of the populations in the Jura Mountains (Switzerland and France).

This scattered distribution in Central Europe results from a strong decrease in European populations. It has disappeared from Austria, the Netherlands (Derron, pers. comm.), from the Polish Tatra Mountains in the 1960s (Mirkowa, pers. comm.) and recently from Germany (Wagner, pers. comm.), eleven stands were lost in Scotland (Welch, 2002), five stands in France (Olivier et al., 1995) and 26 in Switzerland (Käsermann and Moser, 1999). Exploitation, turf extraction and drainage of wetlands are generally considered as the main cause of disappearance (Warncke, 1980; Lockhart, 1989; Welch, 1996; Käsermann and 
Moser, 1999). As a glacial relict, the plant was always rare in Central Europe, and gathering for herbarium is another cause (Käsermann and Moser, 1999). Excessive trampling by cattle (Prost, pers. comm.) and intensive grazing by sheep (Welch, 1996) are sometimes considered as a threat. Despite different studies on the ecological requirements of $S$. hirculus in Europe (e.g. Welch, 1970; Ohlson, 1986, 1988b; Lockhart, 1989; Welch, 1996), none has done soil descriptions and a synthesis is missing for an efficient conservation (Heywood and Iriondo 2003). This is even more necessary now as the present fragmented distribution of the species adds to the risk of local extinction (Lienert et al., 2002).

The last Swiss population, in the Jura Mountains, is very important to conserve, as it is by far the largest in Central Europe. No threat is presently known and the population seems to increase slightly. By its importance and good condition, this population is ideal to study ecological requirements of $S$. hirculus in order to find suitable wetlands for potential reintroductions.

This study aims (1) to synthesise ecological data about $S$. hirculus in Europe, (2) to get a comprehensive understanding of its ecology in the Jura Mountains (plant communities, importance of the microtopography, soil conditions), (3) to suggest management of the remaining populations and to have the necessary knowledge to find wetlands suitable for new introductions.

\section{Material and method}

\subsection{Study sites in the Jura Mountains}

The wetland Sèche de Gimel is located in the Jura Mountains, in western Switzerland $\left(46^{\circ} 33^{\prime}\right.$ $\mathrm{N}, 6^{\circ} 14^{\prime} \mathrm{E}$ ), $1310 \mathrm{~m}$ asl (Fig. 1). Annual rainfall is $1630 \mathrm{~mm}$, with a regular distribution through the year, and about 140 days of snow on the ground. Mean temperature is $3.5^{\circ} \mathrm{C}$, with 120-135 days of vegetation growth. The wetland lays in a small valley within a limestone geological context. Being $750 \mathrm{~m}$ long and $150 \mathrm{~m}$ broad, it offers a large diversity of ecological conditions. It is limited by small limestone cliffs on the north, with basic rich fen at the bottom, and watered by springs from the surroundings pastures on the south-eastern side, with mesotrophic to eutrophic fen close to the mouth. A small raised bog has developed at the south. Different intermediate ecological conditions are met between these poles (Christe et al., 1990). Dolines on the western side and a light slope (0.3 \%) drain the marsh. The site has been visited by botanists for a long time (e.g. Durand and Pittier, 1882), but we do not have any reliable data on the previous abundance of $S$. hirculus. The site is known for other rare postglacial relicts in Central Europe such as Carex heleonastes (Gallandat, 1982) and the bryophytes Paludella squarrosa and Meesia triquetra (Geissler and Zoller, 1978).

The wetland is situated in the middle of wooded pastures, grazed by young bovines between June and September. They generally start to feed in the wetland in July or August, when grass gets shorter in the surrounding meadows. No precise historical data exists about the exploitation of this pasture, but we know that the valley was already grazed in $12^{\text {th }}$ century (Badel-Grau, 1900) and exploitation has never stopped since, with an intensive use of the forests and pastures between the $17^{\text {th }}$ and $19^{\text {th }}$ centuries (Rochat, 1995).

The other Jura populations of $S$. hirculus are in France. Frasne is a poor fen, $830 \mathrm{~m}$ asl, with about 50 to 70 plants (Ferrez et al., 2001, 2004); trampling and grazing were important (Prost, pers. comm), but conservation actions started recently (Dehondt and Ferrez, 2004). Les Pontets is a protected raised bog, $1000 \mathrm{~m}$ asl, with 5-10 plants. Le Bélieu (890 m asl) had a couple of plants, but nobody could find them recently (Dehondt and Ferrez, 2004).

The species composition of the stands in the Jura Mountains were compared with personal or previously published, phytosociological relevés (Braun-Blanquet, 1964). 


\subsection{Hydrology, microtopography and rooting}

Water-level fluctuations in summer were studied with a device of seven piezometers in the wetland Sèche de Gimel, each in an important group of $S$. hirculus. The level was recorded about every week during the growing and flowering time (from 19.5.2004 to 9.8.2004) and once a month later, up to 27.10.2004. An automatic limnigraph recorded continuously water level and temperature (70 cm deep) for two months (26.5.2004-20.7.2004).

This wetland has a complex microtopography, with a succession of small mounds created by mosses (Fig. 2). In order to study the repartition of $S$. hirculus in relation with this topography, six 10 m-transects were sampled through dense populations of $S$. hirculus. Every transect ran next to a piezometer. A 2 m-rigid rod was maintained perfectly horizontal above the ground and we measured every $5 \mathrm{~cm}$ the distance to the ground and recorded the closest species (Fig. 2). When the water table was visible in a hollow, we measured its distance to the rod. This was repeated 5 times for every transect, without changing the height of the rod. The extremities of these transects were permanently marked and will serve for future population monitoring.

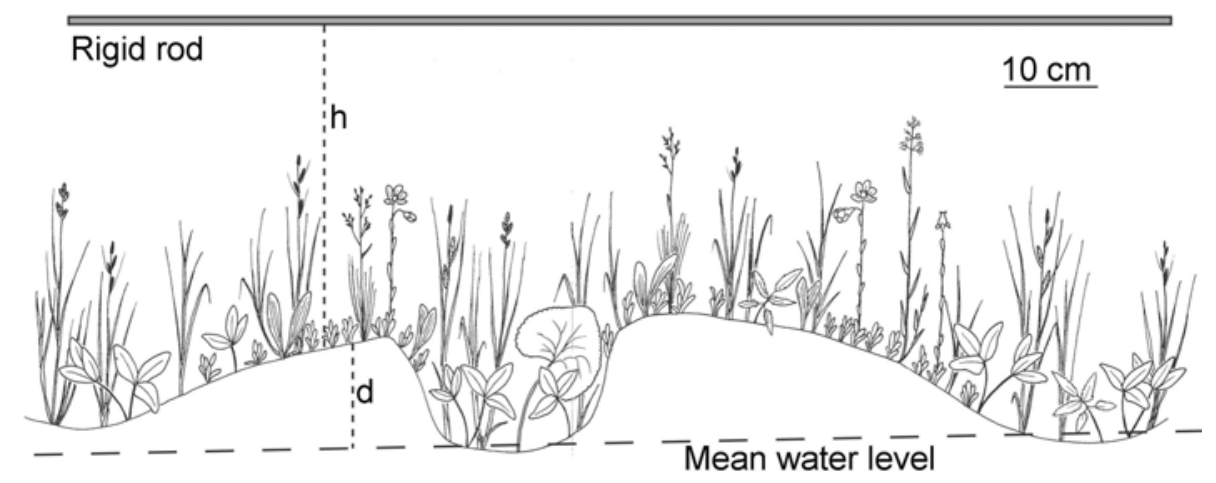

Fig. 2. Microtopography in the wetland, with a cow footprint in the middle of a mound. The height h between the rod and the soil surface was measured in the field. For analyses, distance d between species and mean water level is used. Illustrated species: Agrostis stolonifera, Caltha palustris, Carex diandra, Carex nigra, Carex rostrata, Festuca rubra s.l., Menyanthes trifoliata, Potentilla palustris, Saxifraga hirculus and Swertia perennis.

From the different measures of water level along the transect, a regression line was calculated and corrected to the mean water level measured in the piezometer (we assumed that the water level changed identically along one transect). We used this level as a reference for the transect to calculate the position of every species to the mean water level (Fig. 2). In order to study if the shape of the soil surface influences the distribution of $S$. hirculus, a convexity index was calculated for each point as the difference between its height and the mean height of the previous and next points. This index is positive if the surface is convex, negative if concave and null otherwise.

Two small blocks of peat with a few individuals of $S$. hirculus were cut and cautiously dismantled by avoiding moving the roots to examine the roots position and spread.

\subsection{Soil descriptions}

The soils were described by five auger cores. In the laboratory, each horizon was subject to the following analyses: (1) water content at $105^{\circ} \mathrm{C}$; (2) loss on ignition at $600{ }^{\circ} \mathrm{C}$ (an estimate of the rate of organic matter in peaty soils; Baize and Girard, 1995); (3) granulometric fractionation by wet sieving at 2000, 200, and $50 \mu \mathrm{m}$ (Gobat et al., 1991) to deduce fibres (> $200 \mu \mathrm{m}$ ) content (Bascomb et al., 1977), and thus the types of horizons H (AFES, 1998); (4) water $\mathrm{pH}$, and $\mathrm{KCl} \mathrm{pH}$; (5) index with sodium pyrophosphate: alkaline extraction by $\mathrm{Na}_{4} \mathrm{P}_{2} \mathrm{O}_{7}$ 
and measurement of the absorbance at $550 \mathrm{~nm}$ (an estimate of the degree of humification of the peat; Kaila, 1956).

\subsection{European data and statistical analyses}

From previous articles and personal contacts, we got botanical relevés and ecological data on $S$. hirculus populations from Great Britain, Denmark and Finland. This species was not always the main topic of the research (appendix 1). Species composition of the different European regions was compared with a synoptic table, where each region is represented by one column and species by its frequency in the relevés of the region.

Botanical relevés and species were sorted with cluster analyses in order to look for common and differential species between regions in Europe and between wetlands in the Jura Mountains (software MULVA-5, Wildi and Orloci, 1996, with all vascular species, weighting on frequency or abundance, Van der Maarel coefficient and minimum variance clustering). Bryophytes were eliminated for these analyses, because they were not considered by all the authors, but were added again in the final table.

A synthesis of the ecology of $S$. hirculus was established on the basis of information provided by 21 articles and reports. With the exception of Warncke (1980) and Ohlson (1986), the data are very specific in general. They relate to five fields: hydrology, soil science, climatology, the role of domestic or wild herbivores, as well as that of man. Unfortunately, the heterogeneity of the data does not allow for any statistical comparison.

\section{Results}

\subsection{Ecological and soil descriptors in Europe}

S. hirculus needs sunny conditions (Ruuhijärvi, 1960; Olesen and Warncke, 1990). The air temperature does not seen important, but cold water is more favourable (Gillet, 1982; Ohlson, 1986). Warncke (1980) measures a maximum water temperature between 12 and $14{ }^{\circ} \mathrm{C}$ in August (measurements made $25 \mathrm{~cm}$ under the surface of the water). The macroclimate is fresh and humid, as shown by the area of general distribution.

The influence of cattle is divided into three degrees of intensity. The absence of grazing is considered negatively (Welch and Rawes, 1964; Ohlson, 1989b): the increase in the height and biomass of the vegetation that is no longer grazed, combined with the progressive return of woody plants, decreases light availability (Welch, 1996). A weak to moderate grazing is regarded as positive, by the creation of gaps in the vegetation and the contribution of light up to the surface of the soil (Welch, 1970). On the other hand, intense grazing is considered negatively, either by bovines or sheep (Kelly, pers. comm.; Ferrez et al., 2004). Olesen and Warncke (1990) announced that wild herbivores contribute to dissemination of seeds of $S$. hirculus. The rarity of these mammals could be one of the causes of the reduction in $S$. hirculus.

The soil cover generally consists of bryophytes (Gillet, 1982; Lockhart, 1989; Olesen and Warncke, 1990). S. hirculus roots in a kind of superficial "mossy bed", whose loose structure facilitates the lateral circulation of water.

A water table very close to the surface is a fundamental condition for $S$. hirculus. The soil is described as very wet to saturated (Welch, 1970; Rosskopf, 1971; Gillet, 1982). Warncke (1980) measured the mean depth of the water table as between 3.2 and $10.4 \mathrm{~cm}$ in different spring fens where this species was present and Ohlson (1986) reported a mean water table of $14-20 \mathrm{~cm}$ for the highest density of $S$. hirculus along a gradient. Water is flowing, sometimes weakly, along a small slopes or from a spring (Ruuhijärvi, 1960; Welch, 1970; Gillet, 1982; Lockhart, 1989; Kelly, pers. comm.). This circulation guarantees good oxygenation. Warncke 
(1980) and Ohlson (1986) observed that fluctuations of the water table were low (respectively $7.0-15.5 \mathrm{~cm}$ in different populations and about $15 \mathrm{~cm}$ ).

In these very wet soils, the chemical quality of the water is a good indicator of the edaphic conditions. The $\mathrm{pH}$ levels are neutral or slightly acid (Table 1) and the electric conductivity reflects a broad range between fresh waters typical of the raised bogs, and alkaline waters loaded with $\mathrm{CaCO}_{3}$ (Ruuhijärvi, 1960). There is a large range of concentration for different minerals, especially Ca. S. hirculus appears thus stenoïc compared to the hydric regime and rather euryoïc compared to the chemistry of the water chemistry.

Table 1. Chemical analyses of water, soil and plants in Saxifraga hirculus stands. Values are minimal and maximal values from literature. Extreme values, far outside of the range of the other data, are given in brackets. References are ${ }^{1}$ Welch (1970), ${ }^{2}$ Rosskopf (1971), ${ }^{3}$ Warncke (1980; soils data between 0-25 cm), ${ }^{4}$ Ohlson (1986), ${ }^{5}$ Ohlson (1988b; soils data between 1-6 cm and plant data from the leaves, *for extractable concentrations of the elements), ${ }^{6}$ Lockhart (1989), ${ }^{7}$ Högbom and Ohlson (1991).

\begin{tabular}{|c|c|c|c|c|c|c|}
\hline & \multicolumn{2}{|l|}{ Water } & \multicolumn{2}{|c|}{ Soil } & \multicolumn{2}{|c|}{ Plant } \\
\hline & $\begin{array}{c}\text { Range of values } \\
{[\mathrm{mg} / \mathrm{l}]}\end{array}$ & References & $\begin{array}{c}\text { Range of values } \\
\text { [mg/g dry wt] }\end{array}$ & References & $\begin{array}{c}\text { Range of values } \\
\text { [mg/g dry wt] }\end{array}$ & References \\
\hline $\mathrm{pH}$ & $5.9-7.5$ & 4,6 & $5.8-6.5$ & 2 & & \\
\hline $\mathrm{NO}_{3}-\mathrm{N}$ & $<0.01-0.09(0.68)$ & $1,5,6,7$ & $<0.01^{*}$ & 5 & & \\
\hline $\mathrm{NH}_{4}-\mathrm{N}$ & $0.01-0.11$ & $1,5,7$ & $0.01-0.24^{*}$ & 5 & & \\
\hline $\begin{array}{l}\mathrm{N} \\
\mathrm{PO}_{4}\end{array}$ & $<0.01-0.02$ & 5,7 & $\begin{array}{c}3.4-19.9 \\
<0.01-0.18^{\star}\end{array}$ & $\begin{array}{l}5 \\
5\end{array}$ & $9-19.7$ & 5 \\
\hline $\mathrm{K}$ & $0.1-3.6$ & $1,4,5,6,7$ & $0.1-3.0$ & $3,4,5$ & $20.8-35.6$ & 5 \\
\hline $\mathrm{Ca}$ & $1.5-59.0$ & $1,4,5,6,7$ & $7.7-44.0$ & $3,4,5$ & $15.7-32.4$ & 5 \\
\hline $\mathrm{Mg}$ & $1.4-10.6$ & $1,4,5,6$ & $0.5-2.4$ & $3,4,5$ & $2.3-7.0$ & 5 \\
\hline $\mathrm{Fe}$ & $<0.01-4.5(19.8)$ & $4,5,6$ & $3.4-64(400)$ & 4,5 & $0.1-0.4$ & 5 \\
\hline $\mathrm{Mn}$ & $0.01-2.1$ & 5,6 & $0.03-14.9$ & 3,5 & $0.001-0.002$ & 5 \\
\hline
\end{tabular}

The presence of peat is noted in all localities (e.g. Gillet, 1982; Ohlson, 1986; Lockhart, 1989). Paradoxically, there is no study of its quality (fibres, degree of decomposition, etc). Its depth ranges between 1.5 and 4.5 m (Warncke, 1980; Ohlson, 1986, 1988a), but it does not seem to be determining. The organic matter of the soil is never studied.

The trophic level of the soils (contents of N, P and K) is extremely variable, considered as oligotrophic (Ruuhijärvi, 1960; Welch, 1970) to eutrophic (Ruuhijäri, 1960; Ohlson, 1986), with an optimum given as mesotrophic (Welch, 1970; Gillet, 1982; Ohlson, 1986). This broad range is confirmed by the measured values for soil extractable pools of $\mathrm{NH}_{4}$ and $\mathrm{PO}_{4}($ Table 1). The degree of minerotrophy is in conformity with values for histosols, with ash between 6.6 and $10.4 \%$ (Ohlson, 1986). One can particularly observe a large variability of Ca content, sometimes over short distances (Ohlson, 1986), and an accumulation of $\mathrm{K}$ in the top layer (Damman, 1978; Warncke, 1980; Gobat, 1984; Ohlson, 1988b). The optimum soil pH for $S$. hirculus is uncertain as only one value was published (Table 1).

The quality of the subjacent rock does not play any part, since limestone or crystalline subsoils are found (Warncke, 1980; Gillet, 1982; Lockhart, 1989). However, substrates rich in metals (Fe, Mn, Zn) may be limiting as the species seems to have a low resistance to them. It probably possesses an effective avoidance mechanisms to reduce heavy metal uptake, as shown by very low concentrations of Fe, Mn and Zn in leaves (Table 1; Ohlson, 1988b). 
Table 2. Synoptic table of floristical relevés from different European stands with Saxifraga hirculus. Roman numerals give the frequency of the species in the original table: $\mathrm{r}<10 \%$, I = 11-20\%, II = 21-40 \%, III = 41-60 $\%$, IV $=61-80 \%, \mathrm{~V}=81-100 \%$. Occasional species, never appearing with more than $20 \%$, are not printed. The origin of the data is given in appendix 1.

\begin{tabular}{|c|c|c|c|c|c|}
\hline $\begin{array}{l}\text { Number of relevés } \\
\text { Number of vascular species }\end{array}$ & $\begin{array}{c}\text { Finland } \\
27 \\
64\end{array}$ & $\begin{array}{c}\text { Denmark } \\
100 \\
48\end{array}$ & $\begin{array}{c}\text { Jura } \\
22 \\
78\end{array}$ & $\begin{array}{c}\text { Ireland } \\
8 \\
46\end{array}$ & $\begin{array}{c}\text { Scotland } \\
5 \\
47\end{array}$ \\
\hline \multicolumn{6}{|c|}{ Common and frequent species } \\
\hline Saxifraga hirculus & III & I & $\mathrm{V}$ & $\mathrm{V}$ & $\mathrm{V}$ \\
\hline Epilobium palustre & II & III & IV & IV & $\mathrm{V}$ \\
\hline Carex rostrata & III & II & $\mathrm{V}$ & II & IV \\
\hline Caltha palustris & $\mathrm{r}$ & I & $\mathrm{V}$ & IV & $\mathrm{V}$ \\
\hline Equisetum palustre & II & III & II & II & $\mathrm{V}$ \\
\hline Galium palustre & $\mathrm{r}$ & $r$ & III & IV & III \\
\hline Carex nigra & I & $r$ & IV & 1 & III \\
\hline Angelica sylvestris & $\mathrm{r}$ & $\mathrm{r}$ & II & II & II \\
\hline Cardamine pratensis & & I & III & $\mathrm{V}$ & IV \\
\hline Menyanthes trifoliata & III & IV & III & III & \\
\hline Festuca rubra s.I. & & V & II & II & IV \\
\hline Holcus lanatus & & II & $r$ & $\mathrm{~V}$ & $\mathrm{~V}$ \\
\hline Lychnis flos-cuculi & & I & III & III & $\mathrm{V}$ \\
\hline Equisetum fluviatile & III & I & IV & & II \\
\hline Agrostis canina & & II & II & I & IV \\
\hline Triglochin palustris & & II & $\mathrm{r}$ & II & IV \\
\hline Juncus articulatus & & II & I & II & III \\
\hline "'Calliergonella cuspidata' & & "'III" & "'II" & "V" & "V" \\
\hline Homalothecium nitens & III & II & II & IV & \\
\hline Plagiomnium affine aggr. & $\mathrm{r}$ & $\mathrm{V}$ & II & III & \\
\hline Bryum pseudotriquetrum & II & I & II & II & \\
\hline Drepanocladus vernicosus & II & II & II & 1 & \\
\hline Philonotis fontana & & $r$ & II & II & 1 \\
\hline \multicolumn{6}{|l|}{ Differential species for Finland } \\
\hline Andromeda polifolia & III & $\mathrm{r}$ & & & \\
\hline Carex chordorrhiza & III & & & & \\
\hline Betula pubescens & II & & $\mathrm{r}$ & & \\
\hline Scirpus hudsonianus & II & & & & \\
\hline Salix myrsinites & II & & & & \\
\hline Pyrola rotundifolia & II & & & & \\
\hline Carex lasiocarpa & II & & & & \\
\hline Calamagrostis stricta & II & & & & \\
\hline Betula nana & II & & & & \\
\hline Ćnclidium stygium & Tiٓ & & & & \\
\hline Calliergon richardsonii & II & & & & \\
\hline \multicolumn{6}{|c|}{ Differential species for Denmark } \\
\hline Lotus uliginosus & & $\mathrm{V}$ & & & \\
\hline Mentha aquatica & & IV & $\mathrm{r}$ & & \\
\hline Carex disticha & & IV & & & \\
\hline Carex acutiformis & & III & & & \\
\hline Geum rivale & $\mathrm{r}$ & II & & & \\
\hline Carex flacca & & II & & & \\
\hline \multicolumn{6}{|l|}{ Differential species for Jura } \\
\hline Valeriana dioica & & & $\mathrm{V}$ & & \\
\hline Salix repens & & & III & & \\
\hline Dactylorhiza incarnata & $r$ & & III & & \\
\hline Briza media & & $r$ & II & & \\
\hline Peucedanum palustre & $\mathrm{r}$ & & II & & \\
\hline Myosotis scorpioides & & $r$ & II & & \\
\hline Euphrasia rostkoviana s.l. & & & II & & \\
\hline Polygonum viviparum & & & II & & \\
\hline Swertia perennis & & & II & & \\
\hline \multicolumn{6}{|c|}{ Differential species for British Islands } \\
\hline Luzula multiflora & & $r$ & & II & III \\
\hline Sagina procumbens & & & & II & 1 \\
\hline Ranunculus flammula & & & & II & 1 \\
\hline Montia fontana s.I. & & & & II & 1 \\
\hline Carex echinata & & & & II & i \\
\hline Rumex acetosella & & & & & IV \\
\hline Juncus bulbosus & & & & III & \\
\hline Potamogeton polygonifolius & & & & III & \\
\hline Juncus acutiflorus & & & & II & \\
\hline Stellaria alsine & & & & & II \\
\hline Lophocolea bidentatà & & $\bar{r}$ & & iī & "iiī \\
\hline Hylocomium splendens & & & & 1 & II \\
\hline Pellia sp. & $\mathrm{r}$ & & & & II \\
\hline Riccardia pinguis & & & & III & \\
\hline Cratoneuron filicinum & & & & II & \\
\hline Dicranella palustris & & & & II & \\
\hline Dicranum scoparium & & & & II & \\
\hline Drepanocladus exannulatus & & & & II & \\
\hline Chiloscyphus polyanthos & & & & II & \\
\hline
\end{tabular}


Table 2. (end)

\begin{tabular}{|c|c|c|c|c|c|}
\hline & Finland & Denmark & Jura & Ireland & Scotland \\
\hline Other species & & & & & \\
\hline Galium uliginosum & & IV & IV & & IV \\
\hline Carex diandra & III & & $\mathrm{V}$ & II & \\
\hline Potentilla palustris & I & & $\mathrm{V}$ & III & \\
\hline Cerastium fontanum s.l. & & II & & III & III \\
\hline Anthoxanthum odoratum & & & I & II & $\mathrm{V}$ \\
\hline Carex limosa & II & & III & II & \\
\hline Pedicularis palustris & 1 & & 1 & & $\mathrm{~V}$ \\
\hline Rumex acetosa & & III & II & II & \\
\hline Cirsium palustre & & III & $r$ & & III \\
\hline Carex panicea & & & II & II & II \\
\hline Vaccinium oxycoccos & III & & 1 & II & \\
\hline Carex dioica & III & & II & I & \\
\hline Ranunculus acris s.l. & & $r$ & 1 & & IV \\
\hline Sagina nodosa & & $r$ & 1 & IV & \\
\hline Succisa pratensis & & & III & I & I \\
\hline Parnassia palustris & & $r$ & II & & I \\
\hline Eriophorum angustifolium & $\mathrm{r}$ & & II & & $\mathrm{I}$ \\
\hline Agrostis stolonifera & & & III & III & \\
\hline Trifolium repens & & & II & & III \\
\hline Poa pratensis & II & & III & & \\
\hline Potentilla erecta & & & III & & $\mathrm{I}$ \\
\hline Deschampsia cespitosa s.I. & & & & & II \\
\hline Rhytidiadelphus squarrosus & & آ & & îi & Tाँ \\
\hline Climacium dendroides & $r$ & & II & & IV \\
\hline Aulacomnium palustre & III & & $\mathrm{I}$ & II & \\
\hline Drepanocladus revolvens & II & & II & II & \\
\hline Marchantia polymorpha & & $r$ & 1 & II & \\
\hline Paludella squarrosa & II & 1 & $r$ & & \\
\hline Sphagnum warnstorfii & III & & 1 & & \\
\hline Plagiomnium undulatum & & $r$ & & & III \\
\hline Sphagnum teres & I & & & II & \\
\hline Sphagnum contortum & & & $r$ & II & \\
\hline Occasional vascular specie & 38 & 12 & 27 & 15 & 8 \\
\hline
\end{tabular}

\subsection{Phytosociology}

Data from five European regions were found with precise lists of species (Table 2, appendix 1). All these regions have many species in common growing with $S$. hirculus. Following Oberdorfer (1990), these are mostly species associated to wetlands in general, like Epilobium palustre, to fens (Scheuchzerio-Caricetea fuscae) like Carex nigra, Menyanthes trifoliata, Potentilla palustris or Carex diandra, to reed thicket and big-sedge wetlands (Phragmitetea) like Galium palustre, Equisetum fluviatile, Carex rostrata, or to wet meadows (Molinietalia) like Caltha palustris, Equisetum palustre, Lychnis flos-cuculi, Valeriana dioica or Galium uliginosum. Mosses are mostly ubiquitous species in wet places (Frahm and Frey, 1983), with a preference for base-rich wetlands for Calliergonella cuspidata, Bryum pseudotriquetrum, Homalothecium nitens or Drepanocladus revolvens (Smith, 1978; Geissler pers. comm).

Many species are present only in one European site, generally with a quite low frequency (Table 2). Some have a restricted European distribution (e.g. Salix myrsinites), but most of them are widely distributed and may indicate small differences in the ecological conditions of the wetlands. Finnish data show a trend to raised bogs (Andromeda polifolia, Betula nana, Scirpus hudsonianus), or to poor fen (Carex chordorrhiza, Carex lasiocarpa, Calamagrostis stricta). The influence of agricultural exploitation is visible in Denmark with Lotus uliginosus and Geum rivale, while Great Britain has a few acidophilous species (Rumex acetosella, Carex echinata, Luzula multiflora). 
Table 3. Floristic relevés with Saxifraga hirculus in the Jura Mountains. Abundance indices follow BraunBlanquet (1964), $\mathrm{x}=$ presence without estimation of the cover. Sites: $\mathrm{S}=$ Sèche de Gimel, Switzerland; $\mathrm{F}=$ Frasne, France; B = Le Bélieu, France; $\mathrm{P}=$ Les Pontets, France. The origin of the data is given in appendix 2 and occasional species in appendix 3.

\begin{tabular}{|c|c|c|c|c|c|c|c|c|c|c|c|c|c|c|c|c|c|c|c|c|c|c|}
\hline Releve number & 1 & 2 & 3 & 4 & 5 & 6 & 7 & 8 & 9 & 10 & 11 & 12 & 13 & 14 & 15 & 16 & 17 & 18 & 19 & 20 & 21 & 22 \\
\hline$\overline{\text { Site }}$ & $\mathrm{S}$ & $\mathrm{S}$ & $\mathrm{S}$ & $\mathrm{S}$ & $\mathrm{s}$ & $\mathrm{s}$ & $\mathrm{s}$ & $\mathrm{s}$ & $\mathrm{S}$ & $\mathrm{S}$ & $\mathrm{S}$ & $\mathrm{S}$ & $\mathrm{F}$ & $\mathrm{F}$ & $\mathrm{F}$ & $\mathrm{F}$ & $\mathrm{F}$ & $P$ & $\mathrm{~F}$ & $\mathrm{~F}$ & $\mathrm{~B}$ & $\mathrm{~B}$ \\
\hline Area $\left[\mathrm{m}^{2}\right]$ & & & 2 & 15 & 10 & 16 & 10 & 15 & 30 & 5 & 10 & 3 & & & 100 & 1 & 4 & 4 & 4 & 1 & 2 & 3 \\
\hline Herb cover [\%] & & & 75 & 75 & 85 & 95 & 75 & 75 & 100 & 75 & & & 90 & & 100 & 90 & & 95 & 90 & 70 & 50 & 50 \\
\hline Moss cover [\%] & & & 95 & 95 & 90 & 75 & 90 & 95 & 10 & 100 & & 80 & 100 & & & 100 & & 95 & 95 & 100 & 100 & 100 \\
\hline Number of vascular species & 25 & 24 & 21 & 29 & 21 & 16 & 19 & 16 & 18 & 23 & 20 & 9 & 30 & 38 & 34 & 15 & 16 & 16 & 22 & 20 & 23 & 22 \\
\hline \multicolumn{23}{|l|}{ Common species } \\
\hline Saxifraga hirculus & 2 & 2 & + & 1 & 2 & 2 & 1 & 2 & + & 1 & 1 & 2 & 1 & 1 & 1 & 2 & + & + & + & 1 & + & 1 \\
\hline Potentilla palustris & 1 & + & 2 & 2 & 2 & & 2 & 2 & & 2 & 3 & 1 & 2 & 2 & 2 & 2 & 2 & 1 & 3 & 2 & 2 & 2 \\
\hline Caltha palustris & 2 & 3 & 1 & + & 1 & 2 & 1 & 2 & 2 & 1 & 1 & 2 & 1 & 2 & 2 & 1 & + & & 1 & - & + & 1 \\
\hline Valeriana dioica & 2 & 2 & 1 & + & + & 1 & + & + & 1 & & $r$ & & 1 & 2 & 2 & 1 & + & 2 & + & + & 1 & 1 \\
\hline Carex diandra & + & + & + & 1 & 2 & 2 & 1 & 2 & + & 1 & 1 & & 2 & 2 & & 1 & + & & 1 & 1 & & + \\
\hline Carex rostrata & 2 & 1 & 2 & 2 & 1 & 1 & & 2 & 5 & 2 & 2 & & 3 & 3 & 3 & 2 & 1 & 1 & + & & 1 & \\
\hline Carex nigra & 2 & 3 & 2 & 2 & 2 & 2 & 3 & + & + & 2 & 1 & 2 & 2 & & & & & & & & 1 & 1 \\
\hline Galium uliginosum & 1 & & + & + & + & & + & & & & & & + & 2 & 2 & + & + & $r$ & + & + & + & + \\
\hline Epilobium palustre & 1 & + & & + & 1 & & & & 1 & & 1 & & + & 1 & 1 & + & + & & + & + & + & \\
\hline Equisetum fluviatile & & & $\mathrm{r}$ & + & + & & & & & + & + & & 2 & + & + & 1 & + & & 1 & + & + & + \\
\hline Menyanthes trifoliata & & & + & & + & & r & & & 4 & & & 2 & 1 & & & 1 & + & 3 & 1 & 2 & 2 \\
\hline \multicolumn{22}{|l|}{ 1st group species } & \\
\hline Poa pratensis & + & + & + & + & 1 & + & + & + & + & & + & & + & + & + & & & & & & & \\
\hline Agrostis stolonifera & + & 1 & & + & + & + & + & + & 2 & + & & 1 & 1 & & + & & & & + & & & \\
\hline Lychnis flos-cuculi & + & 1 & + & + & + & 1 & & & & & & & 1 & 1 & 2 & 1 & + & & + & & & \\
\hline Dactylorhiza incarnata & & 1 & + & + & & & + & 1 & & 1 & + & & 1 & + & + & & & & & & & \\
\hline Cardamine pratensis & 1 & 1 & + & + & + & + & & 1 & & + & + & & & & & & & & & & & \\
\hline Swertia perennis & 1 & 1 & 1 & & 1 & 1 & + & & + & & & & & & & & & + & & & & \\
\hline Myosotis scorpioides & 1 & 2 & & + & + & + & & & 1 & & & & & + & + & & & & & & & \\
\hline Festuca rubra s.I. & + & 1 & & + & 1 & + & & & & & & 1 & & & & & & & & & & \\
\hline Rumex acetosa & & & + & + & + & + & & + & & & $\mathrm{r}$ & & & & & & & & & & & \\
\hline Polygonum viviparum & & & & 1 & + & & 2 & + & & 2 & & & & & & & & & & & & \\
\hline Euphrasia rostkoviana s.I. & & & + & 2 & & & 1 & + & & + & & & & & & & & & & & & \\
\hline Briza media & + & 1 & & & & & & & & & & & & + & + & & & & & & & + \\
\hline Blysmus compressus & 1 & + & 2 & 2 & & & & & & & & & & & & & & & & & & \\
\hline Eriophorum latifolium & & + & & $r$ & & & 1 & & & 2 & & & & & & & & & & & & \\
\hline Sagina nodosa & + & + & & & & & & & & & & & + & & & & & & & & & \\
\hline Agrostis capillaris & 1 & 1 & & & & & & & & & + & & & & & & & & & & & \\
\hline Juncus articulatus & & & & + & & & & & & & & & & 2 & 2 & & & & & & & \\
\hline Trifolium pratense & & & & & & & + & & & & & & + & & + & & & & & & & \\
\hline Plagiomnium affine aggr. & & & $x$ & $x$ & $x$ & $x$ & & $x$ & & & & 2 & + & & & & & & & & & \\
\hline Philonotis fontana & & & $x$ & $x$ & $x$ & & & $x$ & & & & & + & & & & & & & & + & \\
\hline Drepanocladus revolvens & & & $x$ & $x$ & $x$ & $x$ & & & & 5 & & + & & & & & & & & & & \\
\hline Calliergonella cuspidata & & & $x$ & $x$ & $x$ & $x$ & & & & & & & 1 & & 1 & & & & & & & \\
\hline Bryum pseudotriquetrum & & & & & & $x$ & & $x$ & & 1 & & + & 1 & & & 1 & & & & & & \\
\hline Drepanocladus vernicosus & & & $x$ & & & & & $x$ & & & & & + & & + & 1 & & & & & & \\
\hline Calliergon giganteum & & & $x$ & & $x$ & $x$ & & & & & & & 1 & & & & & & & & & \\
\hline Marchantia polymorpha & & & & & $x$ & $\mathrm{x}$ & & & & & & & 1 & & & + & & & & & & \\
\hline 3rd group species & & & & & & & & & & & & & & & & & & & & & & \\
\hline Equisetum palustre & & & & & & & & & + & & & & & 1 & 1 & & & & $r$ & 1 & 1 & 1 \\
\hline Agrostis canina & & & & & & & & & & & & & & + & 1 & + & & & & + & 1 & + \\
\hline Vaccinium oxycoccos & & & & & & & & & & & & & + & & & & & & & 4 & 2 & \\
\hline Molinia caerulea & & & & & & & & & & & & & & & & & & $r$ & & & 1 & 3 \\
\hline Viola palustris & & & & & & & & & & & & & & & & & & & & & 1 & + \\
\hline Aulacomnium palustre & & & & & & & & & & & & & + & & & & & & & 4 & 2 & 1 \\
\hline Sphagnum warnstorfii & & & & & & & & & & & & + & & & & & & & & & 4 & 2 \\
\hline Other species & & & & & & & & & & & & & & & & & & & & & & \\
\hline Salix repens & & 1 & & & & & + & & & + & & & 2 & + & + & 1 & 2 & 4 & + & & 1 & \\
\hline Succisa pratensis & & & & + & & & & & & + & + & & & + & + & & & $r$ & + & + & 1 & + \\
\hline Carex limosa & & & & & & & 1 & 2 & & + & + & & & & & 1 & + & + & 2 & + & & \\
\hline Potentilla erecta & + & & + & & & & & & & + & & & + & + & + & & & & & + & + & 2 \\
\hline Carex panicea & 2 & & & & & & + & & & & & & 1 & + & + & & & & & + & + & 1 \\
\hline Eriophorum angustifoliu & & & + & + & & & & & & + & $r$ & & + & & & & + & & & & & + \\
\hline Peucedanum palustre & & & & & & & & & & & & & 2 & + & & 1 & + & & 2 & + & & \\
\hline Carex dioica & + & + & & & & & & & & 1 & & & & & & & & & & 1 & 1 & 1 \\
\hline Parnassia palustris & $r$ & & & + & & & & & & & & + & 1 & + & & & & & & & + & \\
\hline Trifolium repens & & & & + & & & & & & & & & 1 & + & + & 2 & & & 1 & & & \\
\hline Angelica sylvestris & & & & & & & & & & & & & + & + & + & & & & + & 1 & & \\
\hline Anthoxanthum odoratum & & & & & & & & & & & & & & + & + & & & & $r$ & + & & \\
\hline Ranunculus acris friesianus & & & & & & & & & & & & & & 1 & 1 & & & & & + & + & \\
\hline Pedicularis palustris & & & & & & & & & & & & & + & + & & & & & & & + & \\
\hline Polygonum bistorta & & & $r$ & & & & & & + & & & + & & & & & & & & & & \\
\hline "Climacium dendroides & & & n'mumum & & "ntum & & & & & & & & "umum" & & 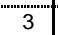 & "umum" & & & & "'t"mut" & & 3 \\
\hline Homalothecium nitens & & & $x$ & $x$ & $x$ & & & & & & & 2 & & & & + & & & & & 1 & + \\
\hline Number of occasional spe & 0 & 0 & 0 & 1 & 0 & 1 & 0 & 0 & 4 & 2 & 2 & 1 & 3 & 9 & 8 & 0 & 0 & 4 & 1 & 0 & 0 & 3 \\
\hline
\end{tabular}


The Jura Mountains data gather twenty-two floristic relevés from the four last populations (Table 3, appendix 2). The analyses divide them in three groups. The first one is mostly from the Sèche de Gimel and is characterised by pastureland species (Molinio-Arrhenatheretea): Lychnis flos-cuculi, Myosotis scorpioides or Cardamine pratensis prefer wet conditions, Poa pratensis, Polygonum viviparum or Festuca rubra s.l. are common in the surrounding pastures. The influence of trampling by cattle is locally important with Agrostis stolonifera and Sagina nodosa, typical species from wet pastures (Oberdorfer, 1990), and four species (Swertia perennis, Blysmus compressus, Eriophorum latifolium, Sagina nodosa) show a preference for base-rich wetlands. The second group, only with French data, is intermediate, lacking most of the characteristic species of the other groups. The third group has the lowest plant cover and the characteristic species indicate a trend to raised bog, with acidophilous species like Agrostis canina, Vaccinium oxycoccos and Sphagnum warnstorfii, and the low abundance of Caltha palustris, Carex rostrata or Carex diandra.

\subsection{Hydrology, microtopography and rooting in Sèche de Gimel}

The mean level of the water table lay between 5.6 and $14.1 \mathrm{~cm}$ in depth, showing a certain homogeneity of general hydrous conditions (Table 4). The populations of S. hirculus were almost never flooded, in spite of a water table potentially very close to the surface (highest levels between $0.5 \mathrm{~cm}$ above soil-level and $5.5 \mathrm{~cm}$ in depth). On the other hand, in the extreme case, the water table went down to $37.5 \mathrm{~cm}$. This lowering was however only of short duration in 2004 (Fig. 3). The uninterrupted measures by the limnigraph confirmed the weak variations of the water table. Rain rather quickly raises its level, which then recedes slowly.

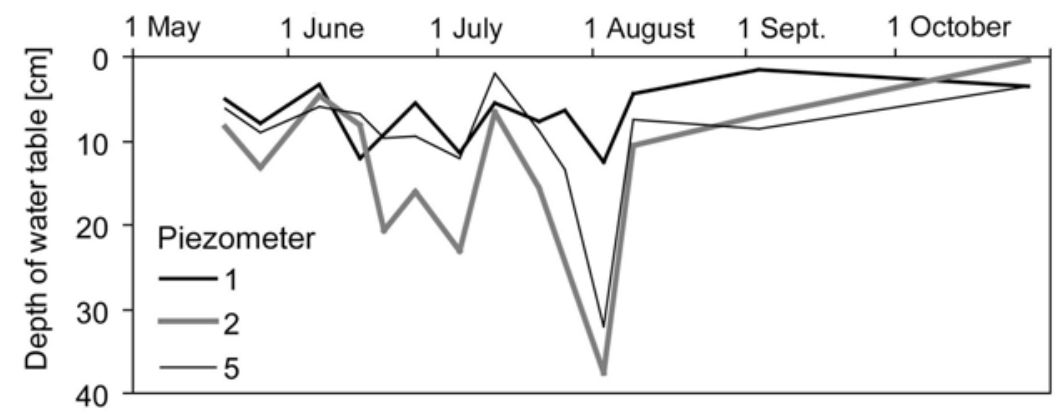

Fig. 3. Fluctuations of water level in 3 piezometers from May to October 2004 in the middle of Saxifraga hirculus populations, Sèche de Gimel, Switzerland (same numbers as Table 3).

The temperature of the water, measured at $70 \mathrm{~cm}$ in depth, increased gradually by 8.6 to 12.6 ${ }^{\circ} \mathrm{C}$ between May 26 and July 9, to stabilize between 12.1 and $12.6{ }^{\circ} \mathrm{C}$ until the end of measurements on July 20, 2004.

The measures along transects indicated that $S$. hirculus has an optimal distance of 8 to $14 \mathrm{~cm}$ from the mean water level (mean $10.9 \pm 3.2 \mathrm{~cm}, n=181$; Fig. 4). However, it is able to grow closer or farther from the water, with extreme limits at 1.3 and $19.0 \mathrm{~cm}$. These values are significantly different form the values for the whole measures along transects (mean $9.5 \pm$ $4.4 \mathrm{~cm}, n=1206, p=0.0001$ with a Wilcoxon Rank Test). The shape of the soil surface influences slightly the distribution of $S$. hirculus as well. Convexity is a little higher for this species (mean $0.24 \pm 0.93, n=181$ ) than for the whole transects (mean $0.01 \pm 1.21, n=$ $1188)$, but the difference is little significant $(p=0.0118)$. The species has a small preference for mounds, probably for the ideal distance to water table that they offer. 


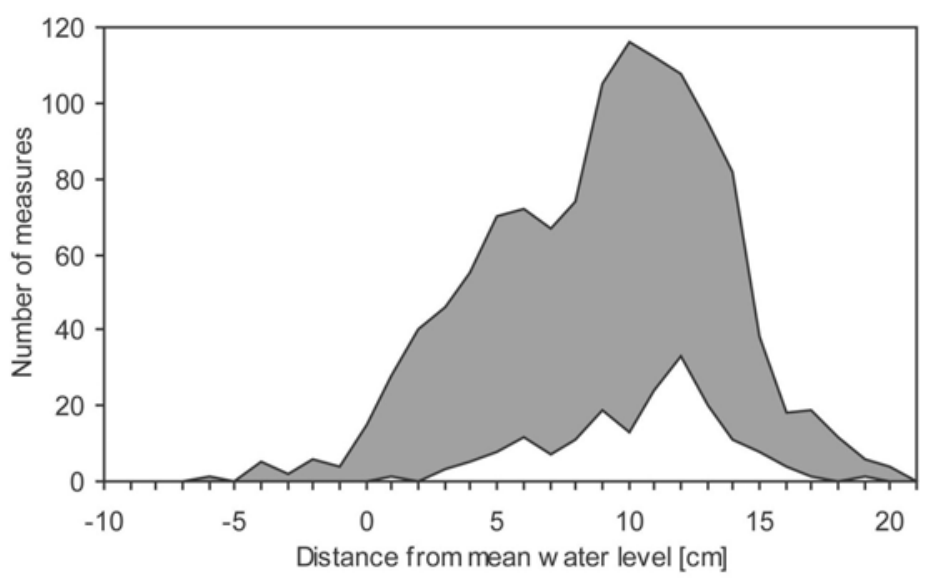

Fig. 4. Frequency of distances measured between soil surface and mean water level along six transects in Sèche de Gimel, Switzerland, with (white, $n=181$ ) or without (grey, $n=1206$ ) Saxifraga hirculus.

Table 4. Highest, lowest and mean level of water table in Sèche de Gimel, Switzerland. Measurements of 7 piezometers from May to October 2004, placed in Saxifraga hirculus populations.

\begin{tabular}{cccc}
\hline Piezometer & $\begin{array}{c}\text { Maxima } \\
{[\mathrm{cm}]}\end{array}$ & $\begin{array}{c}\text { Minima } \\
{[\mathrm{cm}]}\end{array}$ & $\begin{array}{c}\text { Mean } \\
{[\mathrm{cm}]}\end{array}$ \\
\hline 1 & 1.5 & 12.6 & 6.9 \\
2 & 0.5 & 37.5 & 14.0 \\
3 & 5.5 & 31.0 & 12.4 \\
4 & 2.0 & 32.0 & 9.6 \\
5 & 1.5 & 14.9 & 6.7 \\
6 & -0.5 & 27.5 & 5.6 \\
7 & 4.5 & 35.5 & 14.1 \\
Mean & 2.1 & 27.3 & 9.9 \\
\hline
\end{tabular}

Roots of the two samples of $S$. hirculus had a striking horizontal distribution, only $1-3 \mathrm{~cm}$ under the soil surface, set at the limit between living (green) and dead (brown) bryophytes (Fig. 5). Such distribution is frequent by wetland plants, with roots avoiding the water table (Polomski and Kuhn, 1998), but the plants stay most of the time in a wet environment due to water capillarity. Senn-Irlet (pers. comm.) did not find any mycorrhizas on this species.

\subsection{Soil descriptions in Sèche de Gimel}

The five soil samples consisted of fairly thick peat. The horizon $\mathrm{C}$ was only reached once at less than one meter of depth, but the peats were often enriched by clay from 40 to $60 \mathrm{~cm}$ (Table 5). Four soils were characterized by prevalent Hm horizons (mesic histosols) and one by prevalent Hf horizons (fibric histosol). All however comprised horizons of the other type, indicating somewhat changing conditions on the site, but without general direction. Three of the mesic histosols were fibric on the surface, proving current peat formation.

Although very peaty, these soils were different from typical histosols in raised bogs, and in particular from that in Sèche de Gimel which is thicker and formed of Sphagnum peat for more than 2 meters in depth (Wegmüller, 1966). Several characters reflect their ecotonal situation between the peat bog itself and the fen, or the surrounding meadows (Table 5): apparently illogical superposition of horizons with peat of different quality (bryophytes, sedges, rhizomes), illustrating the "moving" soil conditions of this zone of transition; deep horizons mixed with clay within horizons of anmoor, which is typical of soils located on the edge of peat bogs in the Jura Mountains (Gobat, 1984); fibres content does not decrease gradually with the depth, as often observed in stable raised bogs; pyrophosphate index of biochemical decomposition is on average higher than that of the acid peat bogs; water $\mathrm{pH}$ is 
neutral to slightly alkaline; $\mathrm{KCl} \mathrm{pH}$ is slightly acidic, translating the strong proportion of organic acids of the peat, rather well humified (cf. pyrophosphate index).

Table 5. Soil description and analyses from 5 profiles of peat in Sèche de Gimel, Switzerland.

\begin{tabular}{|c|c|c|c|c|c|c|c|c|}
\hline Horizon & $\begin{array}{l}\text { Depth } \\
{[\mathrm{cm}]}\end{array}$ & Field description & $\begin{array}{c}\text { Water } \\
\text { content [\%] }\end{array}$ & $\begin{array}{c}\text { Loss on } \\
\text { ignition } \\
{[\%]}\end{array}$ & $\begin{array}{c}\text { Fibres } \\
\text { content } \\
{[\%]}\end{array}$ & $\begin{array}{l}\text { Pyro } \\
\text { index }\end{array}$ & $\mathrm{pH} \mathrm{H} \mathrm{H}_{2} \mathrm{O}$ & $\mathrm{pH} \mathrm{KCl}$ \\
\hline $\mathrm{Hf2}$ & 18 & Idem. Roots 2/5, from $10 \mathrm{~cm}$ & 88.5 & 84.1 & 56.5 & 16 & 7.5 & 5.8 \\
\hline Hf3 & 37 & Reddish-brown peat, closer, with some looser pockets. No roots & 85.7 & 82.2 & 65.9 & 18 & 7.2 & 5.6 \\
\hline $\mathrm{Hm} 2$ & 96 & Reddish-brown peat with mineral material (clay). Heavy (slimy) touch & 79.6 & 53.1 & 31.3 & 66 & 7.5 & 5.6 \\
\hline Hf1 & 5 & $\begin{array}{l}\text { Light brown-yellowish dead mosses, very loose. Blackish sheathes } \\
\text { from Carex rostrata and C. diandra. Relatively clear limit }\end{array}$ & 90.9 & 90.8 & nd & 8 & 6.8 & 6.5 \\
\hline $\mathrm{Hf} 2$ & 21 & $\begin{array}{l}\text { Very fibrous, black, loose peat. Roots } 3 / 5 \text {. Many rhizomes from } \\
\text { Carex }\end{array}$ & 92.4 & 92.1 & 90.1 & 12 & 6.9 & 6.2 \\
\hline $\mathrm{Hf} 4$ & 79 & Black, fibrous peat & nd & nd & nd & 53 & 6.5 & 5.4 \\
\hline $\mathrm{HmCg}$ & 90 & Mix of peat and brown-beige silt & 75.9 & 43.6 & 23.3 & 74 & 7.2 & 5.3 \\
\hline Cca & $>98$ & Yellow-beige silt and sand. Macrorests of shiny twigs (from Betula?) & nd & nd & nd & nd & 8.1 & 6.9 \\
\hline Hf1 & 5 & $\begin{array}{l}\text { Fibres from dead bryophytes. Very numerous rootlets. Porous peat, } \\
\text { but maintained by fibres. Clear limit }\end{array}$ & 92.3 & 73.3 & 46.5 & 23 & 8.1 & 6.8 \\
\hline $\mathrm{Hf} 2$ & 24 & $\begin{array}{l}\text { Black, loose and very porous peat. Small quantities of mineral } \\
\text { material (silt, clay) }\end{array}$ & 89.5 & 82.8 & 56.0 & 16 & 7.2 & 5.7 \\
\hline $\mathrm{Hm} 1$ & 39 & Mesic, loose, brown-black peat. More mineral material & 81.4 & 48.8 & 29.4 & 28 & 7.2 & 5.4 \\
\hline $\mathrm{Hm} 2$ & 79 & $\begin{array}{l}\text { High mineralised peat, with much fine material. Brown-black with } \\
\text { more fibrous reddish-brown pockets }\end{array}$ & 67.2 & 37.2 & 20.2 & 55 & 7.2 & 5.0 \\
\hline $\mathrm{Hm} 1$ & 75 & Dark brown peat, more or less loose, with fine material. Vague limit & 88.2 & 73.5 & 32.0 & 35 & 7.1 & 5.4 \\
\hline $\mathrm{Hm} 2$ & $>99$ & Idem, but more compact. Some light-browner spots & 86.7 & 70.4 & 30.8 & 33 & 7.1 & 5.5 \\
\hline $\mathrm{Hm1}$ & 6 & Reddish-brown peat with fine roots $4 / 5$. Clear limit & 87.0 & 55.5 & 35.7 & 22 & 8.2 & 7.0 \\
\hline $\mathrm{Hf}$ & 22 & Idem, but a bit looser. Brown, then black. Presence of fine material & 89.3 & 83.4 & 61.7 & 22 & 6.9 & 5.5 \\
\hline $\mathrm{Hm} 2$ & 59 & Dark brown, loose peat. More fine material. Vague limit & 90.0 & 78.6 & 38.0 & 27 & 7.3 & 5.9 \\
\hline AnHm1 & 79 & Peat-rich, pasty anmoor. Vague limit & 77.5 & 26.7 & 32.0 & 56 & 7.2 & 5.6 \\
\hline AnHm2 & $>98$ & Anmoor with reddish-brown blacky peat & 81.5 & 25.6 & 36.0 & 43 & 6.5 & 5.3 \\
\hline
\end{tabular}

\section{Discussion}

\subsection{Ecological conditions and competition}

The data collected in the literature and our results give a more precise picture of the ideal ecological conditions for Saxifraga hirculus. The species frequently found with S. hirculus (Table 2 and 3) indicate together wet to very wet conditions, a mesotrophic soil, neutral to slightly acid, with some species which prefer base-rich water (Oberdorfer, 1990). The sometimes-encountered heterogeneous composition, a mixture of wetland and pastures species, can be explained by the influence of the frequent grazing (Denmark, Scotland, Switzerland) and the microtopography giving very wet and drier conditions only a few centimetres apart.

Roots and growth conditions indicate that a good availability of oxygen is an important factor. Although growing in a wet environment, with a stable water table close to the soil surface, $S$.

Vittoz et al. (2006)

Biological Conservation 131: 594-608 
hirculus is sensitive to water and develops its roots in order to avoid it (Fig. 5). It grows in loose, mesic or more often fibric peat, with running water, generally along springs water (Ruuhijärvi, 1960; Warncke, 1980; Ohlson, 1986) or close to the drains in the wetland Sèche de Gimel (Vittoz, 1997). This water circulation assures a good oxygen supply, and its relatively low temperature $\left(12-14{ }^{\circ} \mathrm{C}\right.$; Warncke, 1980 and the present study) implies a high oxygen content. According to Larcher (2003), oxygen solubilization at $10^{\circ} \mathrm{C}$ attains $356 \mu \mathrm{M}$, and only $291 \mu \mathrm{M}$ at $20^{\circ} \mathrm{C}$. Moreover, the loosely structured peat may contribute to a constant, high water table by shrinking in dry periods (de Mars et al., 1997).

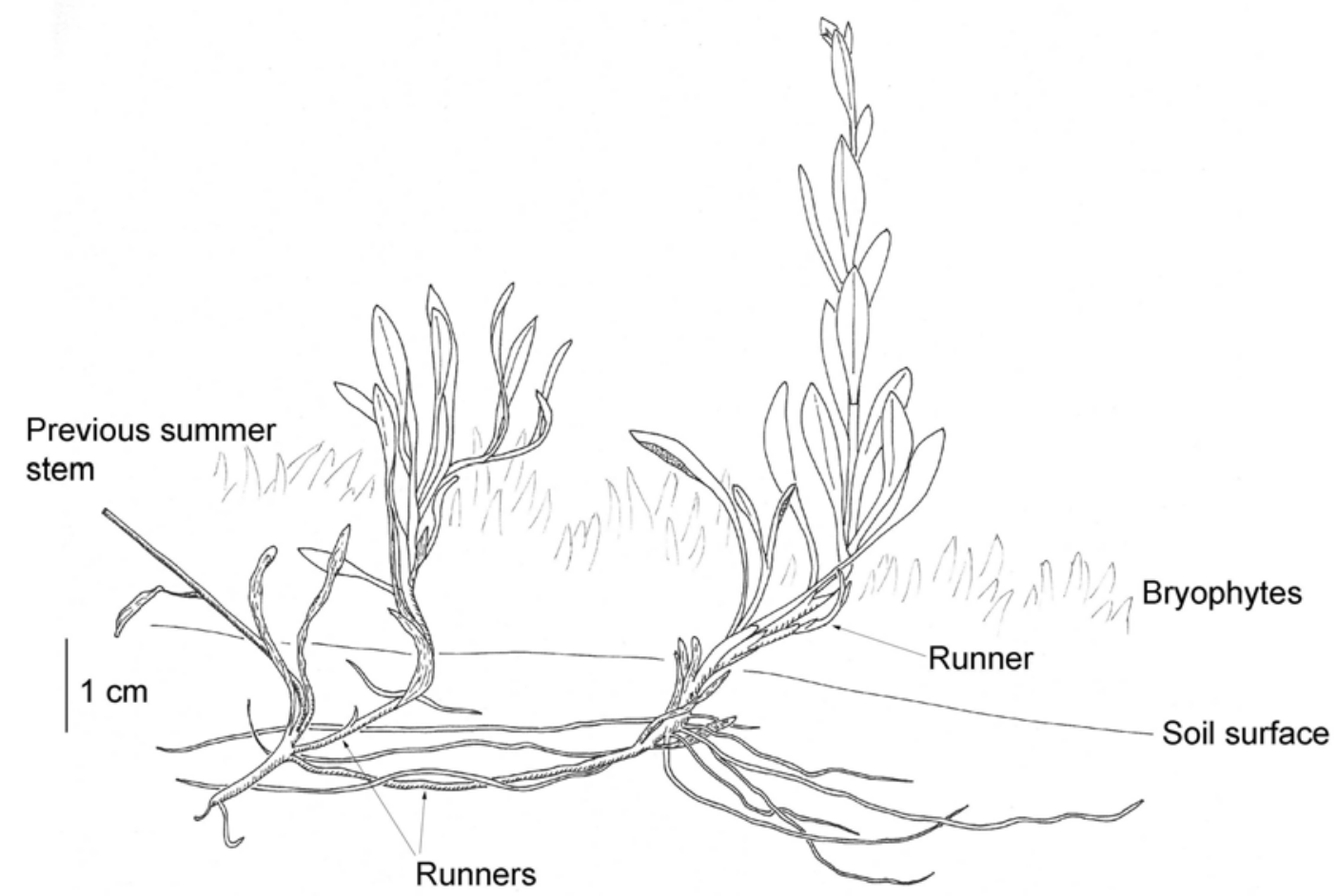

Fig. 5. Root position of Saxifraga hirculus in a wetland, Sèche de Gimel, Switzerland. The stem of previous summer produced two runners. One is developing a flowering stem (right) and a new runner.

S. hirculus is mainly a weak competitive species growing generally in grass layer with open cover (Table 3, Rosskopf, 1971). Olesen and Warncke (1990) showed that seed germination was favoured by high light intensity. In a fence experiment, the species disappears after the end of grazing because of the dense and high surrounding grasses (Welch and Rawes, 1964) and a reduction of flowering ramets was observed in habitats characterized by expansion of woody species after abandonment of haymaking and grazing (Ohlson, 1989b). Its small leaves, only a few centimetres above the ground, are unable to compete in fens with dense Carex populations. Thus, the saxifrage finds its good conditions on bryophytes carpets, 8 to $14 \mathrm{~cm}$ above the mean water table (Fig. 4; Warncke, 1980; Ohlson, 1986). If the water table is lower (except during short periods), the competition with other species increases. If the water table is higher, it is submerged and dies (Welch, 2002), or competition with Sphagnum is enhanced (Grosvernier et al., 1997). Seedlings seem particularly sensitive to the risks of drought or submersion (Welch, pers. comm). The optimal conditions can be maintained only with small fluctuations of the water level, probably lower than $\pm 10 \mathrm{~cm}$ around the mean. These conditions are frequent along streams depending on small springs (Ohlson, 1986), but are less common in wetlands depending on a water table. Low summer temperatures and frequent precipitations like in the Jura Mountains, Great Britain and Scandinavia (Walter and 
Lieth, 1967) avoid long droughts, and an effective evacuation of water from the wetland (light slope) is important to avoid long flooding.

This weak ability of $S$. hirculus to compete with other species is visible in its nutrient status as well. It grows in a wide range of $\mathrm{N}, \mathrm{P}$ and $\mathrm{K}$ conditions, although there is no correlation between the concentration of these nutrients in plants and in peat (Table 1; Ohlson, 1988b). It means that it has a good ability to regulate its nutrient concentration in changing conditions but it is probably not able to take advantage of rich conditions for a better growth. Wassen et al. (2005) showed that this species is one of the few endangered wetland plants to grow mostly in N-limited conditions. We can hypothesize that $S$. hirculus development among sedges like $C$. rostrata is facilitated in conditions with nitrogen deficit: Carex rostrata growth is limited when $S$. hirculus takes advantage of its higher capacity to use nitrate as source of nitrogen (Hogböm and Ohlson, 1991). Conversely, with higher nitrogen availability, the saxifrage may be outcompeted for light by taller species.

Spring fens with peat seem to produce the optimal ecological conditions for S. hirculus and offer stable conditions (Warncke, 1980). Most of North European populations grow in such fens. However, they are very rare in Central Europe mountains. The slopes are generally too steep, and the calcium content of the water is too high in limestone context (Jura and large parts of the Alps) to have peat accumulation. Tufa formation (mineral accumulation with specific bryophytes) is thus much more common. The rare ideal situations have a very limited extent among grazed pastures and have been degraded by trampling for a long time. The saxifrage has to find similar conditions in wetlands to survive in Central Europe.

\subsection{Basic fens dynamics in the Jura Mountains and cattle influence}

In the humid conditions of the Jura Mountains, wetland has a natural dynamics from rich fen to raised bogs, which may be unfavourable to Saxifraga hirculus and could force its extinction. Gillet (1982) described in the Jura Mountains a plant community, SphagnoCaricetum dioicae, as the transition between the basic rich fens and the raised bogs, with three stages of development: (1) bryophytes low mounds (e.g. Homalothecium nitens, Climacium dendroides, Aulacomnium palustre, Drepanocladus revolvens), with a $\mathrm{pH}$ around 6; (2) colonization of the mounds by basic fens peat moss (Sphagnum teres, S. warnstrofii) which raise and enlarge the mounds and decrease nutrients and $\mathrm{pH}$ below 5; (3) the mounds merge each other and colonization of raised bog peat moss (Sphagnum magellanicum), with a $\mathrm{pH}$ around 3.5. A similar succession was observed in Poland by Wassen and Joosten (1996). $S$. hirculus has its optimum in the first stage of the development, before the colonization by peat moss (Gillet, 1982), that it seems to avoid. In Sèche de Gimel, it rarely grows in Sphagnum patches, although they are common, and it has recently disappeared from Le Bélieu, where it grew in Sphagnum warnstorfii stands (relevés 21 and 22 in Table 3; Dehondt and Ferrez, 2004). Ohlson's results (1986) also show that $S$. hirculus is less common when $S$. warnstorfii dominates the moss layer. The low $\mathrm{pH}$ and the competition for nutrients may be responsible, but Welch (2002) observed S. warnstorfii overtopping young transplanted rosettes of saxifrage. Clymo and Reddaway (1974) measured a 2-3 cm year ${ }^{-1}$ growth for Sphagnum stems, enough to overtop and shade young rosettes of S. hirculus (Welch, 2002). The low resistance of $S$. hirculus to heavy metals may be another reason to explain its decrease in low pH-conditions (Ohlson, 1988b).

Different factors may limit the development of competitive species and minimise peat formation. One of these is the good oxygenation of the water which allows considerable biological activity and thus slows down peat formation. In the wetland Sèche de Gimel, cattle are certainly important. They graze a part of the competitive grasses (Welch and Rawes, 1964; Ausden et al., 2005) and the trampling regularly packs the soil surface and limits Sphagnum growth (Fig. 2; Arnesen, 1999; Wahren et al., 2001). This stops the natural succession to raised bog. The saxifrage may locally suffer damages, but the runners help to 
repair them and allow the colonization of the newly restored areas. Moreover, the disturbed areas (bare peat) are favourable for seedlings (Ohlson, 1986, 1989a), and Olesen and Warncke (1990) suggested that dispersion is naturally made by large mammals (e.g. deer, buffalo) when they wallow in grass or unwillingly eat the seeds. However, with a high level of grazing, disturbances are too severe for the saxifrage: trampling damages are higher than recolonization, grazing of flowers before maturity impends sexual reproduction (Ferrez et al., 2004), destruction of bryiophytes' bed limits its growth and there is a increased competition with species favoured by cowpats or mineralization of bare peat which liberates nitrate. This delicate balance between cattle grazing and Saxifraga development seems to be visible in palynological data from Sèche de Gimel (Sjögren, in press): its presence increased around AD 1100 in a period of increasing use of pasturelands but decreased around AD 1700, perhaps suffering from augmentation of cattle numbers and deforestation, possibly with draining of the mire. After this last change, the peat became more decomposed.

These results suggest changing conditions in the wetland through the centuries under natural or anthropic influences. Peat profiles showed a succession of different qualities (Table 5), and microtopography fluctuations were already observed in other peat bogs (Tüxen, 1976): mounds grow and disappear, changing the growth conditions for plants. It is probable that $S$. hirculus has been moving on the wetland for a long time, and will carry on as long as changes are not quicker than its ability to colonize new sites. We can hypothesize that peat development or too quick changes were responsible for the extinction of the species in some still-existing wetlands.

\subsection{Conservation of Saxifraga hirculus and restoration of populations}

S. hirculus has a narrow niche-breadth, and the potential sites for its growth are very limited in Central Europe. However, an adequate management may maintain, improve or create ideal conditions for this species. We suggest four types: cattle grazing, mowing, re-introduction and substrate management.

In the wetland Sèche de Gimel, cattle grazing conserved $S$. hirculus. The pressure must be appropriately chosen. If it remains weak to moderate, the pasture does not receive too much nitrogen from the faeces and trampling damage is limited. We unfortunately lack historical data in Sèche de Gimel or experiments in other places to estimate the adequate pressure. Presently, about 40 calves and 65 heifers are grazing 141 hectares of pastures in summer (but milk cows grazed previously). This corresponds to about 0.5 adult bovine-unit ha ${ }^{-1}$ for 140 days every year. However the wetlands represent only $8.5 \%$ of the total area. The pressure on the wetland may temporally increase in dry years, when available grass is less sufficient in the surroundings pastures, as observed in Frasne in 2003 (Ferrez et al., 2004). The conditions seems to be adequate in Sèche de Gimel where a high density population is observed in a site regularly used by cattle and a high genetic diversity was observed in the whole population (Freymond, 1999).

The mowing of wetlands seems to be another way to preserve $S$. hirculus population. Ohlson (1986) suggested that one Swedish population increased in a period when haymacking eliminated ligneous species and hummock-forming mosses (Sphagnum sp.) from a fen, and its occurrences often declined where formerly-mown marshlands were abandoned (Ohlson, 1989b).

Successful trials in botanical gardens proved that re-introduction is possible. Capsules are ripe in September in the Jura Mountains, seeds germinate easily (Olesen and Warncke, 1990; Welch, 2002), although depending on their origin (Ohlson, 1989a). Germination is possible after pre-chilling of seeds in refrigerator during winter and use of a mixture of peat or compost with siliceous sand and gravel (Moret, pers. comm.). In Central Europe, the simultaneous presence of Potentilla palustris, Caltha palustris, Valeriana dioica, Menyanthes trifoliata, Carex diandra, Carex rostrata and Carex nigra may help to recognize potential 
sites. But other characteristics described above are important as well. Indoor germination and transplant of seedlings were made in Scotland with a quite good success (Welch, 2002).

Finally, it would also be necessary to consider the management of the soil substrate. It is a question of supporting ecotonal situations in which a growth of fibrous peat is effective (water table close to the surface), but where it is slowed down by constraints such as a neutral $\mathrm{pH}$, no Sphagnum species, or a moderate decomposition of the peat due to well oxygenated water. This "pedological" management is particularly interesting in two cases: (1) an "intermediate" phytosociological context (poor fen from Caricion lasiocarpae for example), where it is necessary to take care of the maintenance of current conditions, or to even try to extend them laterally; (2) a revitalization of edges of peat bogs (former pits of exploitation), where it would often be necessary to recreate a water current, if too stagnant in the ditches. Such management has never been tested as far as we know.

None of these four types of management guarantees, alone, the conservation of the species. The ideal is to be able to combine several of them according to local conditions. It is also obviously necessary that the general ecological context remains favourable (climate, neighbouring vegetation, human pressure, etc).

\section{Conclusion}

This research integrates original results with literature and gives the most complete portrait, as far as we know, of the ecology of Saxifraga hirculus. It is thus a solid basis for its conservation in Central Europe. The phytosociological and pedological study in Sèche de Gimel (Swiss Jura Mountains), in comparison with the descriptions of other European populations, showed that this wetland presents very good conditions to conserve this species.

S. hirculus needs a low competition from sedges and peat bog mosses to grow. This can be achieved in natural conditions by a stable water table close to the soil surface, with running, cold water, a neutral to slightly acid $\mathrm{pH}$, and a nitrogen deficit. It disappeared mainly following the destruction of its habitats, but natural succession may be unfavourable as well. In many populations, especially in Central Europe, its survival seems to depend on a moderate grazing or mowing to limit competition. However, sites with appropriate ecological conditions are rare. An optimal substrate management, based on present knowledge, could improve the conditions in little adequate situations and facilitate introduction.

Future researches around $S$. hirculus ought to improve knowledge on the influences of cattle grazing (optimal pressure), to select adequate sites for re-introduction and to monitor the large Swiss population in order to react early enough if problems arise. Climate change and nitrogen atmospheric pollution, which may increase competition of surrounding species or allows the colonization of new competitive species, could be a new challenge for $S$. hirculus conservation.

\section{Acknowledgment}

We are very grateful to P. Geissler who identified mosses in Sèche de Gimel, B. Senn-Irlet who looked for mycorrhizas, A. Fragnière for analyses of soil samples, P. Perrochet and F. Bossy in Centre d'hydrogéologie (University of Neuchâtel) who lent the limnigraph. We benefited from discussions with several botanists, including D. Welch, M. Derron, H. Olde Venterink who read and gave us valuable comments on the manuscript. Thank you to J. Grant and D. Welch for partial translation and English verification. We are grateful to Service des forêts, de la faune et de la nature (Canton de Vaud) and the village council of Gimel for protecting Saxifraga hirculus and field work authorizations. This study was supported by Lausanne and Neuchâtel Universities. 


\section{Appendixes}

Appendix 1. Origin of the data in Table 2.

Data are country, reference with the table and aim of the study.

Finland, Ruuhijärvi (1960), tab. 23, rel. 1-27, vegetation of wetlands. Denmark, Warncke (1980), tab. 16, spring fen. Jura, tab. 3 of this article, ecology of Saxifraga hirculus. Ireland, Lockart (1989), tab. 1, rel. 1-8, new stands of S. hirculus. Scotland, Welch (1996), tab. 2, rel. B1, T1, B2, T2, C1, vegetation with S. hirculus.

\section{Appendix 2. Origin of the relevés in Table 3.}

Data are reference with the number of the relevé if published or author and year otherwise and location.

Rel. 1. Gallandat (1982), tab. 12, rel. 7, Sèche de Gimel; 2. Gallandat (1982), tab. 12, rel. 8, Sèche de Gimel; 3. Vittoz, 2001, Sèche de Gimel; 4. Vittoz, 2001, Sèche de Gimel; 5. Vittoz, 2001, Sèche de Gimel; 6. Vittoz, 2001, Sèche de Gimel; 7. Vittoz, 1996, Sèche de Gimel; 8. Vittoz, 2001, Sèche de Gimel; 9. Vittoz, 1995, Sèche de Gimel; 10. Gillet (1982), rel.4, Sèche de Gimel; 11. Richard, 1972 (field notebook 48), Sèche de Gimel; 12. Geissler \& Zoller (1978), tab. 1, rel. 16, Sèche de Gimel; 13. Royer et al. (1979), tab. VIII, rel. 77, Frasne; 14. Gallandat (1982). tab. 16, rel. 2, Frasne; 15. Braun-Blanquet, 1946 (field notebook "Jura and Langenthal 1946"), Frasne; 16. Gillet (1982), rel. 5, Frasne; 17. Moret, 1996, Frasne; 18. Vittoz \& Moret, 1996, Les Pontets; 19. Vittoz, 1996, Frasne; 20. Gillet (1982), rel. 6, Frasne; 21. Gillet (1982), rel. 8, Le Bélieu;. 22. Gillet (1982), rel. 7, Le Bélieu. Relevés 1 and 2 were originally classified in Caricetum fuscae, rel. 10, 16, 20, 21 and 22 in Sphagno warnstorfii-Caricetum dioicae saxifragetosum, rel. 12 in Sphagno warnstorfii-Caricetum nigrae and rel. 13 and 14 in Caricetum diandrae.

\section{Appendix 3. Occasional species of Table 3.}

Data are name of the species, number of the relevé and abundance in brackets.

Betula pubescens 18(+); Brachythecium rivulare 5(x); Carex flava 10(1), 14(1); Cirsium palustre 14(+), 15(+); Crepis paludosa 12(+); Dianthus superbus 18(+), 22(+); Eleocharis palustris 11(+), 14(+); Eleocharis quinqueflora 4(2); Eurhynchium striatum 3(x); Filipendula ulmaria 9(+); Holcus lanatus 14(+), 15(+); Juncus alpinus 13(1); Knautia dipsacifolia 18(+); Linum catharticum 14(+), 15(+); Lotus corniculatus 22(+); Lysimachia nummularia 14(+), 15(+); Meesia triquetra 10(1); Mentha aquatica 15(+), 19(+); Mentha arvensis 14(+); Paludella squarrosa 3(x), 12(3); Phalaris arundinacea 11(r); Poa trivialis 6(2), 9(1); Prunella vulgaris 15(+); Ranunculus lingua 13(+); Rhynchostegium riparioides 3(x); Rumex arifolius 9(r); Salix cinerea 14(+), 15(+); Sanguisorba officinalis 22(1); Senecio helenitis 14(+), 15(+); Sphagnum contortum 21(+); Scirpus cespitosus 10(1); Triglochin palustris 13(+); Vaccinium uliginosum 18(2); Veronica beccabunga 9(+).

\section{Bibliography}

AFES, 1998. A sound reference base for soils. The "Référentiel pédologique". INRA, Paris.

Arnesen. T., 1999. Vegetation dynamics following trampling in rich fen at Solendet, Central Norway; a 15 year study of recovery. Nordic Journal of Botany 19, 313-327.

Ausden, M., Hall, M., Pearson, P., Strudwick, T., 2005. The effects of cattle grazing on tallherb fen vegetation and molluscs. Biological Conservation 122, 317-326.

Badel-Grau, J.-L., 1900. Longirod et ses environs, précédé de quelques notes historiques sur le décanat d'Aubonne. Kündig, Genève.

Baize, D., Girard, M.-C., 1995. Référentiel pédologique. INRA, Paris.

Bascomb, C.L., Banfield, C.F., Bruton, G.O., 1977. Characterization of peaty materials from organic soils (Histosols) in England and Wales. Geoderma 19, 131-147.

Boscaiu, N., Coldea, G., Horeanu, C., 1994. The red list of extinct, threatened, vulnerable and rare vascular plants from Romania Flora. Ocrotirea naturii si mediului inconjurator 38, 45-56.

Braun-Blanquet, J., 1964. Pflanzensoziologie. Grundzüge der Vegetationskunde. 3rd edn. Springer, Wien. 
Christe, P., Glaizot, O., Delarze, R., Hainard, P., 1990. La végétation du marais des Amburnex (Jura vaudois, Suisse). Bulletin de la Société Vaudoise des Sciences Naturelles 80, 127-139.

Clymo, R.S., Reddaway, E.F.J., 1974. Growth rate of Sphagnum rubellum in Pennine blanket bog. Journal of Ecology 62, 191-196.

Dahlgaard, J., Warncke, E., 1994. Seed set and germination in crosses within and between two geographically isolated small populations of Saxifraga hirculus in Denmark. Nordic Journal of Botany 15, 337-341.

Damman, A.W.H., 1978. Distribution and movement of elements in ombrotrophic peat bogs. Oikos 30, 480-495.

Dehondt, F., Ferrez, Y., 2004. Connaissance de la flore rare ou menacée de Franche-Comté, Saxifraga hirculus L. Conservatoire Botanique de Franche-Comté, Besançon, France.

de Mars, H., Wassen, M.J., Olde Venterink, H., 1997. Flooding and groundwater dynamics in fens in eastern Poland. Journal of Vegetation Science 8, 319-328.

Durand, T., Pittier, H. 1882. Catalogue de la flore vaudoise. Rouge et Cie, Lausanne.

Ferrez, Y., Prost, J.-F., André, M., Carteron, M., Millet, P., Piguet, A., Vadam, J.-C. 2001. Atlas des plantes rares ou protégées de Franche-Comté. Société d'Horticulture du Doubs et des Amis du Jardin botanique, Turriers, Naturalia Publications, Besançon.

Ferrez, Y., André, M., Morcrette, P., 2004. Suivi de la flore dans le Bassin du Drugeon (Doubs). Principaux résultats de la saison de terrain 2003. Nouvelles Archives Flore jurassienne 2, 25-32.

Frahm, J.-P., Frey, W., 1983. Moosflora. Ulmer Verlag, Stuttgart.

Freymond, H., 1999. Spatial genetic structure in two populations of a rare species: Saxifraga hirculus L. in Switzerland and Denmark. Diplom thesis, University of Lausanne, Switzerland.

Gallandat, J.-D., 1982. Prairies marécageuses du Haut-Jura. Beiträge für Geobotanischen Landesaufnahme der Schweiz 58.

Geissler, P., Zoller, H., 1978. Paludella squarrosa (Hedw.) Brid. an der Südwestgrenze ihrer Verbreitung, Charakterart einer neuen Assoziation des Sphagno-Tomenthypnion Dahl. Candollea 33, 299-319.

Gillet, F., 1982. L'alliance du Sphagno-Tomenthypnion dans le Jura. Documents phytosociologiques N.S. 6, 155-180.

Gobat, J.-M., 1984. Ecologie des contacts entre tourbières acides et marais alcalins dans le Haut-Jura suisse. PhD thesis, Université de Neuchâtel, Switzerland.

Gobat, J.-M., Grosvernier, P., Matthey, Y., Buttler, A. 1991. Un triangle granulométrique pour les tourbes: analyse semi-automatique et représentation graphique. Science du sol 29, 23-35.

Grosvernier, P., Matthey, W., Buttler, A., 1997. Growth potential of three Sphagnum species in relation to water level and peat properties with implications for their restoration in cut-over bogs. Journal of Applied Ecology 34, 471-483.

Hedberg, O.K., 1992. Taxonomic differentiation in Saxifraga hirculus L. (Saxifragaceae) - a circumpolar Arctic-Boreal species of Central Asiatic origin. Botanical Journal of the Linnean Society 109, 337-393.

Heywood, V.H., Iriondo, J.M., 2003. Plant conservation: old problems, new perspectives. Biological Conservation 113, 321-335. 
Högbom, L., Ohlson, M., 1991. Nitrate assimilation in coexisting vascular plants in mire and swamp forest habitats in Central Sweden. Oecologia 87:495-499.

Kaila, A., 1956. Determination of the degree of humification in peat samples. Maatalous Ackakaoushiva 28, 18-35.

Käsermann, C., Moser, D.M., 1999. Fiches pratiques pour la conservation. Plantes à fleurs et fougères. L'environnement pratique, OFEFP, Berne.

Larcher, W., 2003. Physiological Plant Ecology. Springer, Berlin.

Lienert, J., Fischer, M., Diemer, M., 2002. Local extinctions of the wetland specialist Swertia perennis L. (Gentianaceae) in Switzerland: a revisitation study based on herbarium. Biological Conservation 103, 65-76.

Lockhart, N.D., 1989. Three new localities for Saxifraga hirculus L. in Ireland. Irish Naturalist's Journal 23, 65-69.

NBN Gateway. National Biodiversity Network gateway internet page: http://www.searchnbn.net

Oberdorfer, E., 1990. Pflanzensoziologische Excursionsflora. Ulmer, Stuttgart.

Ohlson, M., 1986. Reproductive differentiation in Saxifraga hirculus population along an environmental gradient on a central Swedish mire. Holarctic Ecology 9, 205-213.

Ohlson, M., 1988a. Size-dependent reproductive effort in three populations of Saxifraga hirculus in Sweden. Journal of Ecology 76, 1007-1016.

Ohlson, M., 1988b. Variation in tissue element concentration in mire plants over a range of sites. Holarctic Ecology 11, 267-279.

Ohlson, M., 1989a. Ecotypic differentiation and phenotypic plasticity in Saxifraga hirculus populations in central and northern Sweden. Holarctic Ecology 12, 46-53.

Ohlson, M., 1989b. Dynamik i nord- och mellansvenska populationer av myrbräcka. Svensk Botanisk Tidskrift 83, 1-11.

Olesen, J.M., Warncke, E., 1989. Predation and potential transfer of pollen in a population of Saxifraga hirculus. Holarctic Ecology 12, 87-95.

Olesen, J.M., Warncke, E., 1990. Morphological, phenological and biochemical differentiation in relation to gene flow in a population of Saxifraga hirculus. Sommerfeltia 11, 159-171.

Olivier, L., Galland, J.-P., Maurin, M. (Eds.), 1995. Liste rouge de la flore menace de France. Tome I: espèces prioritaires. Museum d'histoire naturelle, Paris.

Polomski, J., Kuhn, N., 1998. Wurzelsysteme. WSL, Birmensdorf, Haupt, Bern.

Rochat, A., 1995. La commune du Lieu, ses églises, ses cloches et ses forêts. Etudes et documents, Le Pèlerin, Les Charbonnières.

Rosskopf, B., 1971. Pflanzengesellschaften der Talmoore an der Schwarzen und Weissen Laber im Oberpfälzer Jura. PhD thesis, Universität Erlangen-Nürnberg.

Royer, J.-M., Gallandat, J.-D., Gillet, F., Vadam, J.-C., 1979. Sur la présence de groupements relictuels d'affinités boréoarctiques au niveau des marais tremblants (Scheuchzerietalia) du Jura franco-suisse. Documents phytosociologiques N.S. 4, 1085-1092.

Ruuhijärvi, R., 1960. Über die regionale Einteilung der Nordfinnischen Moore. Annales Botanici Societatis Zoologicae Botanicae Fennicae 'Vanamo' 31.

Sjögren, P., in press. The development of pasture woodland in SW Swiss Jura Mountains over 2000 years, based on three adjacent peat profiles. The Holocene.

Vittoz et al. (2006)

Biological Conservation 131: 594-608 
Smith, A.J.E., 1978. The moss flora of Britain and Ireland. Cambridge University Press, Cambridge.

Stenberg, L., Mossberg, B., 2003. Den nya Nordiska Floran. Wahlström \& Widstrand, Stockholm.

Tutin, T.G., Heywood, V.H., Burges, N.A., Moore, D.M., Valentine, D.H., Walters, S.M., Webb, D.A., 1964-1980. Flora Europaea. Cambridge University Press, Cambridge.

Tüxen, J., 1976. Über die Regeneration von Hochmooren. Telma 6, 219-230.

Vittoz, P., 1997. Sèche de Gimel. Bas-marais d'importance nationale n 1486 (Commune du Chenit). Plan de protection et de gestion. Conservation de la Nature, Etat de Vaud, StSulpice, Switzerland.

Wahren, C.H.A., Williams, R.J., Papst, W.A., 2001. Vegetation change and ecological processes in alpine and subalpine Sphagnum bogs of the Bogong High Plains, Victoria, Australia. Arctic Antarctic and Alpine Research 33, 357-368.

Walter, H., Lieth, H., 1967. Klimadiagramm-Weltatlas. Gustav Fischer, Jena.

Warncke, E., 1980. Spring areas: ecology, vegetation, and comments on similarity coefficients applied to plant communities. Holarctic Ecology 3, 233-308.

Warncke, E., Terndrup, U., Michelsen, V., Erhardt, A., 1993. Flower visitors to Saxifraga hirculus in Switzerland and Denmark, a comparative study. Botanica Helvetica 103, 141-147.

Wassen, M.J., Joosten, J.J., 1996. In search of a hydrological explanation for vegetation changes along a fen gradient in the Biebrza Upper Basin (Poland). Vegetatio 124, 191209.

Wassen, M.J., Olde Venterink, H., Lapshina, E.D., Tanneberger, F. 2005. Endangered plants persist under phosphosrus limitation. Nature 437, 547-550.

Wegmüller, S., 1966. Über die spät- und postglaziale Vegetationsgeschichte des südwestlichen Jura. Beiträge für Geobotanischen Landesaufnahme der Schweiz 48.

Welch, D., 1970. Saxifraga hirculus L. in north-east Scotland. Transactions of the Botanical Society of Edinburgh 41, 27-30.

Welch, D., 1996. Habitat preferences and status of Saxifraga hirculus L. in North-East Scotland. Botanical Journal of Scotland 48, 177-186.

Welch, D., 2002. The establishment of recovery sites for Saxifraga hirculus L. in NE Scotland. Botanical Journal of Scotland 54, 75-88.

Welch, D., Rawes, M., 1964. The early effects of excluding sheep from high-level grasslands in the North Pennines. Journal of Applied Ecology 1, 281-300.

Wildi, O., Orloci, L., 1996. Numerical exploration of community patterns. A guide to the use of MULVA-5. SPB Academic Publishing, Amsterdam. 